Documentation et bibliothèques

DOCUMENTATION BIBLIOTHEQUES

\title{
La disponibilité universelle des publications : un nouveau programme de coopération internationale prolongeant le Contrôle bibliographique universel
}

\section{Claire Renaud-Frigon}

Volume 25, numéro 1, mars 1979

URI : https://id.erudit.org/iderudit/1054369ar

DOI : https://doi.org/10.7202/1054369ar

Aller au sommaire du numéro

Éditeur(s)

Association pour l'avancement des sciences et des techniques de la documentation (ASTED)

ISSN

0315-2340 (imprimé)

2291-8949 (numérique)

Découvrir la revue

Citer cet article

Renaud-Frigon, C. (1979). La disponibilité universelle des publications : un nouveau programme de coopération internationale prolongeant le Contrôle bibliographique universel. Documentation et bibliothèques, 25(1), 33-41. https://doi.org/10.7202/1054369ar

\section{Résumé de l'article}

La Fédération internationale des associations de bibliothécaires et des bibliothèques (IFLA) vient d'adopter un programme de Disponibilité universelle des publications (UAP). L'UAP prolonge tout en l'enrichissant le Contrôle bibliographique universel (CBU), puisqu'il place la notion de l'utilisateur au coeur même de l'élaboration du système envisagé. L'article présente le concept et résume cette nouvelle initiative de l’IFLA. Toutes les divisions de la Fédération travaillent à la réalisation de ce programme qui vise à rendre les publications du monde entier accessibles à tous, grâce surtout à de vastes collections et à un réseau de prêt universel. Ce programme ne pourra se réaliser qu'avec la coopération de l'ensemble des bibliothèques de tous les pays.
Tous droits réservés (c) Association pour l'avancement des sciences et des techniques de la documentation (ASTED), 1979
Ce document est protégé par la loi sur le droit d'auteur. L'utilisation des services d'Érudit (y compris la reproduction) est assujettie à sa politique d'utilisation que vous pouvez consulter en ligne.

https://apropos.erudit.org/fr/usagers/politique-dutilisation/ 


\title{
La disponibilité universelle des publications: un nouveau programme de coopération internationale prolongeant le Contrôle bibliographique universel
}

\author{
Claire Renaud-Frigon \\ Centre de documentation sur les bibliothèques \\ Bibliothèque nationale du Canada \\ Ottawa
}

La Fédération internationale des associations de bibliothécaires et des bibliothèques (IFLA) vient d'adopter un programme de Disponibilité universelle des publications (UAP). L'UAP prolonge tout en l'enrichissant le Contrôle bibliographique universel (CBU), puisqu'il place la notion de l'utilisateur au coeur même de l'élaboration du système envisagé. L'article présente le concept et résume cette nouvelle initiative de l'IFLA. Toutes les divisions de la Fédération travaillent à la réalisation de ce programme qui vise à rendre les publications du monde entier accessibles à tous, grâce surtout à de vastes collections et à un réseau de prêt universel. Ce programme ne pourra se réaliser qu'avec la coopération de l'ensemble des bibliothèques de tous les pays.

Recently, the International Federation of Library Associations (IFLA) adopted a program called Universal Availability of Publications (UAP). This program extends and enriches that of Universal Bibliographic Control (UBC), because it places the notion of the user at the very heart of the proposed system. This article presents this concept and details this new initiative of IFLA. All the divisions of the federation are working towards the realisation of this program which aims at rendering all the world's publications available to everyone through vast collections and a worldwide loan network. This program can only be realised with the cooperation of all the libraries of all countries.

La Federación internacional de asociaciones de bibliotecarios y bibliotecas (IFLA) acaba de adoptar un programa de Disponibilidad universal de las publicaciones (UAP). El UAP prolonga el Control bibliográfico universal (CBU) y lo mejora, pues la noción de usuario está al centro del desarrollo del sistema. El artículo presenta el concepto y resume esta nueva iniciativa de la IFLA. Todas las divisiones de la Federación participan a la realización de este programa, cuyo objetivo es poner las publicaciones a la disposición del mundo entero, gracias sobre todo a colecciones importantes y a un sistema universal de préstamo. El programa sólo podrá realizarse con la cooperación de las bibliotecas de todos los países.

Peu de bibliothécaires nord-américains ont eu l'occasion d'assister à un congrès annuel de l'IFLA (Fédération internationale des associations de bibliothécaires et des bibliothèques/International Federation of Library Associations and Institutions). Néanmoins, aucun spécialiste de la documentation ne peut se permettre d'ignorer le travail que la Fédération a accompli depuis plus de vingt ans dans le domaine de la normalisation et de la coopération internationale.
Depuis 1971, le concept de Contrôle bibliographique universel (CBU) sert de point de convergence aux nombreuses activités entreprises par les divisions de I'IFLA et constitue la principale contribution de la Fédération aux programmes de développement entrepris par d'autres organismes, tels l'Unesco, la Fédération internationale de documentation (FID) et le Conseil international des archives (CIA). Dans le rapport qu'elle soumettait au congrès annuel à Grenoble, en août 1973, 
la Secrétaire générale de l'IFLA présentait le concept du Contrôle bibliographique universel en ces termes:

C'est sous ce titre que la FIAB a adopté comme objectif majeur la promotion d'un système universel d'échanges d'informations bibliographiques. Ce système a pour but de fournir, partout et rapidement, sous une forme internationalement acceptable, les renseignements bibliographiques essentiels relatifs aux publications monographiques publiées dans tous les pays. Le projet présuppose la création d'un réseau de centres nationaux se chargeant d'une vaste gamme d'activités ayant toutes rapport à l'édition et aux bibliothèques, activités qui seraient intégrées sur le plan international de manière à former un système universel ${ }^{1}$.

Une nouvelle orientation vient maintenant approfondir et compléter le programme du CBU. II s'agit de I'UAP (Universal Availability of Publications ou Disponibilité universelle des publications), deuxième volet, à moyen terme, du programme de I'IFLA. Comme le soulignait D.J. Urquhart au $4^{\mathrm{e}}$ congrès annuel de la Fédération, tenu du 27 août au 2 septembre 1978 à Strbské Pleso en Tchécoslovaquie, cette nouvelle perspective donnée aux activités de I'IFLA devrait intéresser tous ceux qui oeuvrent dans le domaine de la documentation et rejoindre une préoccupation fondamentale de la profession:

II ne sert à rien aux utilisateurs de découvrir l'existence de documents qui pourraient les intéresser s'ils ne peuvent pas les consulter. Nous arrivons donc à I'UAP - l'accès universel aux publications. II s'agit, en fait, de réexaminer un fort ancien but de notre profession - celui de fournir au lecteur les documents qu'il lui faut ${ }^{2}$.

1. Margreet Wijnstroom, Rapport d'activités, juillet 1972 - juillet 1973, présenté par le Secrétaire général au $39^{\circ}$ congrès de l'IFLA, Grenoble, 1973, p. 4 (112/F/PLE/8 - 115F).

2. D.J. Urquhart, L'UAP: quelles mesures faut-il prendre? Communication présentée au $44^{\mathrm{e}}$ congrès de I'IFLA, Strbské Pleso, 1978, p. 1 (5/CODL/1F).

\section{Aperçu chronologique: du CBU à I'UAP}

1971 Article de Franz Georg Kaltwasser dans le Bulletin de l'Unesco à l'intention des bibliothèques, vol. 25 , no 5 , septembre-octobre 1971, traçant dans ce domaine la voie à I'IFLA pour les années à venir:

Le contrôle bibliographique universel est un projet ambitieux qui peut contribuer à la gestion rationnelle de tout un ensemble d'informations, à savoir celles que fournissent le catalogage des livres et, éventuellement leur indexage. II importe de mettre à la disposition des bibliothèques et des milieux scientifiques une méthode moderne de régulation pour qu'ils puissent faire face à la multiplication de ces informations. Bien entendu, les idées exposées ci-dessus ne pourront être efficaces que dans la mesure où elles se refléteront dans un très grand nombre de mesures concrètes ${ }^{3}$.

$19733^{3}$ congrès annuel de l'IFLA, à Grenoble, du 25 août au $1^{\text {er }}$ septembre. Le thème général portait sur le CBU, système mondial d'échange de renseignements bibliographiques. Des représentants de l'Unesco, de la FID, du CIA et de I'ISO participaient à ce congrès.

1974 Le $1^{\text {er }}$ juillet, création du Bureau international de I'IFLA pour le Contrôle bibliographique universel, à la British Library Reference Division, à Londres. C'était le premier pas concret vers la réalisation du programme de I'IFLA.

1974 Conférence sur la planification des infrastructures nationales en matière de documentation, de bibliothèques et d'archives, du 23 au 27 septembre.

3. Franz Georg Kaltwasser, «Le Contrôle bibliographique universel», Bulletin de l'Unesco à l'intention des bibliothèques, vol. 25 , no 5 (septembre-octobre 1971), 276. 
Organisée par l'Unesco, avec le concours de la FID, de I'IFLA et du CIA, la conférence a étudié la planification des services nationaux d'information (NATIS).

1974 Conférence générale de l'Unesco, du 17 octobre au 23 novembre. Le CBU est accepté, constituant une partie importante du programme de planification des infrastructures nationales (NATIS):

Objectif no 12 - Le Contrôle bibliographique universel: le principe du contrôle bibliographique (CBU) suppose la création préalable d'un contrôle bibliographique national dans chaque pays, garantissant l'enregistrement bibliographique de chaque publication nouvelle au moment de sa parution ${ }^{4}$.

1975 Du 14 au 16 mai, une réunion d'experts sur l'élaboration d'un plan d'action pour le CBU s'est tenue à l'Unesco. Il a été décidé de donner la priorité aux projets visant à encourager l'établissement et le développement des bibliographies nationales.

1975 Etablissement du Bureau de I'IFLA pour le prêt international, à la British Library Lending Division, à Boston Spa. Le Bureau jouera un rôle prépondérant dans le développement de I'UAP et dans la réalisation des objectifs du programme.

1975 L'UAP est inscrit au Programme à moyen terme de I'IFLA pour 19751980. Le projet est présenté comme document de travail au $41^{\mathrm{e}}$ congrès annuel de la Fédération, à Oslo, du 11 au 16 août, sous le titre "Accès universel aux documents»:

4. Unesco, Systèmes nationaux d'information (NATIS); objectifs d'une action nationale et internationale, présenté à la Conférence intergouvernementale sur la planification des infrastructures nationales en matière de documentation, de bibliothèques et d'archives, Paris, 1974, p. 27 (COM74/NATIS/3).
On ne pourra réaliser pleinement les bénéfices du CBU que grâce à la mise à la disposition de tous de la littérature enregistrée. C'est là aussi un objectif auquel la FIAB peut apporter une contribution importante ${ }^{5}$.

1976 L'élaboration du programme UAP et les méthodes pour sa mise en place sont discutées par le Bureau professionnel.

$197743^{e}$ congrès annuel de l'IFLA, à Bruxelles, du 3 au 10 septembre. Plusieurs communications ont porté sur le CBU et les membres de la Section de prêt ont discuté l'UAP.

1977 Congrès international sur les bibliographies nationales, du 12 au 15 septembre, patronné conjointement par l'Unesco et le Bureau international pour le CBU. Les participants sont arrivés à un accord sur la normalisation des descriptions bibliographiques, sur les documents à répertorier, la présentation des bibliographies nationales imprimées et ont discuté de l'échange des notices. Ils ont défini «le cadre applicable à l'agence bibliographique nationale». Ainsi, en assurant le contrôle bibliographique national, les pays font un premier pas vers une plus grande accessibilité des documents recensés.

1977 Le Bureau exécutif de l'IFLA décide que le Bureau pour I'UAP ne sera créé que lorsque le problème des sources de financement sera réglé. Le Groupe de travail sur I'UAP continuera à élaborer le programme, de concert avec le Bureau pour le prêt international, et agira en tant que comité de direction provisoire.

1978 44e congrès annuel de l'IFLA, à Strbské Pleso, en Tchécoslovaquie, du 27 août au 2 septembre. Le thème

5. Fédération internationale des associations de bibliothécaires, Projet de programme à moyen terme, 1975-1980. Document de travail pour le 41e congrès de l'IFLA, Oslo, 1975, p. 25. 
portait sur l'Accessibilité des publications, avec comme sous-thème le Rôle du livre et des bibliothèques dans le développement de la coopération internationale scientifique et culturelle.

1978 Le Projet de programme et de budget pour 1979/80 de I'Unesco recommande l'adoption du CBU/UAP en tant qu'élément du Programme général d'information et plus particulièrement dans le cadre de l'objectif 10.1 portant sur le développement et la promotion des systèmes et services d'information aux niveaux national, régional et international.

1981 Une conférence intergouvernemenou tale sur I'UAP sera organisée par 1982 l'Unesco avec le concours de l'IFLA.

\section{Le concept}

Comme le concept de I'UAP découle du CBU et le prolonge, l'on ne peut se surprendre que ce soit Maurice Line, directeur du Bureau de l'IFLA pour le prêt international, qui ait été l'un des premiers à expliquer la disponibilité universelle des publications. Reprenant l'expression que Dorothy Anderson avait employée quelques années plus tôt pour décrire le CBU, Line affirme que l'UAP est à la fois «un concept, un système et un objectif ${ }^{6}$.

En tant que complément au Contrôle bibliographique universel, I'UAP tentera de favoriser la mise en place de mécanismes visant à rendre accessibles à tous les publications nationales et étrangères, pour lesquelles l'usager aura déjà obtenu une notice bibliographique. II faut noter ici en passant qu'il y a encore flottement dans le choix de l'expression française traduisant le concept UAP. Selon que le concept est envisagé du point de vue de l'usager ou du document, l'on dira "accès universel aux publications» ou "disponibilité universelle des publications». Ce flottement dans la terminologie suffit à souligner l'ampleur du projet et la diversité des activités à planifier.

6. Maurice B. Line, «Disponibilité universelle des publications", Bulletin de l'Unesco à l'intention des bibliothèques, vol. 31, no 3 (mai-juin 1977), 161.
Ce vaste concept vise à assurer la disponibilité des ouvrages identifiés et désirés en donnant à l'utilisateur la possibilité de les obtenir, soit dans leur forme originale, soit sous forme de photocopie, par don, échange ou prêt. Ce qu'il faut souligner avant tout, c'est que le concept de I'UAP ne se limite pas à l'amélioration des services de prêt, mais qu'il s'ouvre sur la perspective d'une satisfaction totale de l'usager:

\begin{abstract}
Puisque le vrai but est la transmission de l'information à l'utilisateur, il faut envisager un système complet englobant l'utilisateur. II faut poser la question: est-ce que l'utilisateur reçoit ce qu'il cherche dans un délai utile? ${ }^{7}$
\end{abstract}

Cette préoccupation fondamentale peut se traduire par un large éventail d'activités, depuis l'étude de l'emplacement d'une bibliothèque ou de l'accès physique à un centre de documentation, jusqu'aux enquêtes sur les catégories de non-utilisateurs, les habitudes de fréquentation, la disponibilité des ressources et le taux de satisfaction des usagers. Les moyens prônés en vue d'arriver au véritable accès aux publications devront être axés sur la création de services adéquats et sur l'utilisation maximale des ressources, puisque ce n'est pas le nombre de volumes achetés qui compte le plus mais le niveau de satisfaction de la clientèle à desservir. Dans la perspective de I'UAP, il faudra repenser certains aspects du développement des collections et du prêt entre bibliothèques, peut-être même changer l'attitude de certains bibliothécaires:

... le rapport annuel de la bibliothèque cesserait dorénavant de faire ressortir les acquisitions et l'amélioration des catalogues. Au contraire, le rapport de la bibliothèque consacrerait son attention principale aux efforts de découvrir (sic) les causes des échecs du service offert au public et les mesures qui auront été prises ou qui seront à prendre pour y remédier ${ }^{8}$.

7. D.J. Urquhart, L'UAP .., p. 14.

8. Ibid., 11. 
II s'agit, on le voit, d'un concept dont l'impact ne saurait être minimisé, dont on entendra parler dans les années à venir et qui pourrait contribuer à la revalorisation des professionnels de la documentation, tant auprès des administrateurs et des politiciens qu'auprès des chercheurs et des citoyens.

\section{Le programme de I'IFLA}

Selon le programme de la Fédération, les résultats du CBU ne pourront atteindre leur véritable dimension que par la disponibilité des publications identifiées. Le contrôle et l'échange de données bibliographiques relatives aux publications parues dans tous les pays préparent l'accès universel aux publications, le document identifié et situé pouvant être obtenu par échange ou prêt. Chaque pays, en principe, a la responsabilité de fournir les publications produites sur son territoire, en prêtant l'original ou en faisant des photocopies pour les autres pays qui en font la demande.

L'UAP ou DUP est un programme «très vaste qui touche au dépôt légal et aux autres modes d'acquisition, à la coordination des bibliothèques par l'intermédiaire des catalogues collectifs par exemple, à l'organisation des services de bibliothèque assurant le libre accès aux ouvrages, aux services de prêt inter-bibliothèque et de photocopie, à l'échange de publications et au rôle des bibliothèques ou centres nationaux. ${ }^{9}$

Selon Maurice Line, le programme UAP implique à la fois que toutes les publications du monde soient accessibles et que tout le monde ait accès aux publications. II faut donc établir de vastes collections et créer un réseau de prêt universel. Le programme à moyen terme, 1975-1980, ratifié à Oslo en 1975, expose quatre volets de l'action de l'IFLA en vue de remplir ces deux conditions essentielles: constitution des fonds, échange de publications, accès aux fonds et droits d'auteur.

9. Maurice B. Line, «Disponibilité universelle...». 161.
La Fédération s'engage premièrement à encourager chaque pays à constituer un centre chargé de recueillir des exemplaires de toutes les publications produites dans le pays, y compris ce qu'il est convenu d'appeler "la littérature souterraine», d'en assurer la préservation et de coopérer à l'intérieur d'un réseau mondial de centres d'échange de publications. Deuxièmement, elle désire faciliter la disponibilité des publications au moyen d'un réseau international de centres nationaux pour le prêt entre bibliothèques et devra faire connaître le point de vue des bibliothèques et des centres de documentation en ce qui concerne le droit d'auteur et la reproduction par photocopie en vue du prêt.

Le programme de I'IFLA s'applique à tous les pays, à tous les genres de bibliothèques et à toutes les catégories de publications et, par le fait même, a des implications au niveau d'une trentaine de sections réunies sous huit divisions. Puisqu'il n'existe pas encore de centre international pour l'UAP, c'est surtout le Bureau de l'IFLA pour le prêt international et un comité provisoire qui sont chargés de faire avancer la Fédération vers la réalisation de ses objectifs.

La Division des bibliothèques de recherche générale s'intéresse surtout au dépôt légal en tant que moyen privilégié permettant de constituer une collection des publications produites dans un pays. La division s'intéresse aussi aux moyens à prendre pour rendre disponibles les publications nationales, par le biais du prêt ou de la photocopie. La Section des bibliothèques nationales discutera aussi de la planification des services d'information et de bibliothèque, afin de s'assurer que la disponibilité des publications et le prêt entre bibliothèques seront traités comme des éléments essentiels à toute politique nationale. De plus, la question d'un développement rationnel des collections à l'intérieur d'un pays, l'achat de publications étrangères et la disponibilité accrue par l'utilisation des catalogues collectifs sont autant de sujets à étudier. La Section des bibliothèques parlementaires s'intéresse tout particulièrement à l'échange des publications officielles. 
La Division des bibliothèques spécialisées pourra étudier les problèmes particuliers aux bibliothèques administratives, aux cartothèques ou aux autres types de bibliothèques qui la composent.

La Division des bibliothèques servant le public en général accorde beaucoup d'importance à l'UAP puisque ce sont les bibliothèques publiques qui doivent mettre l'information à la portée du public. Les bibliothèques publiques doivent aussi renseigner les gens que ne peuvent rejoindre les mécanismes habituels: les handicapés, les malades hospitalisés, les prisonniers, les minorités linguistiques et ethniques. Cette division pourra aussi s'occuper de l'alphabétisation et de l'éducation des adultes.

La Division du contrôle bibliographique traite des rapports entre le CBU et I'UAP, du lien qui existe entre le contrôle bibliographique et la disponibilité d'un ouvrage, des liens avec le NATIS, du dépôt légal et de l'impact de l'automatisation sur la mise à jour des catalogues collectifs et sur les systèmes de prêt.

La Division des collections et services aura un rôle important à jouer dans l'étude des implications de l'UAP, puisqu'elle regroupe la Section des échanges de publications, la Section du prêt entre bibliothèques, celles des publications officielles, des publications en série et des livres rares. Elle s'attaquera au problème de l'échange des publications en tant que procédé permettant aux bibliothèques de se procurer des publications qui ne seraient pas disponibles autrement. Les statistiques des grandes bibliothèques montrent clairement que l'échange des publications officielles et des publications de sociétés savantes reste un facteur important dans le développement des collections. La question maintes fois abordée des centres nationaux de prêt fera l'objet de nombreuses études dans divers pays, puisque les méthodes normalisées faciliteraient l'accès aux publications. L'IFLA s'attachera à définir les genres de documents qui pourront être prêtés et à étudier comment les autres pourront être mis à la disposition des usagers qui en ont besoin. De plus, il faudra que les membres de la
Fédération présentent le plus tôt possible leurs points de vue respectifs sur les lois concernant le droit d'auteur. II faudra même élaborer des listes de base pour que les pays en voie de développement puissent identifier les publications à acheter ou à emprunter.

La Division de l'administration et de la technologie, pour sa part, devra insister sur l'adoption de méthodes statistiques aptes à mesurer le progrès de I'UAP et coordonner les efforts en ce sens avec ceux de I'Unesco. Les récentes découvertes technologiques, comme la reproduction par téléfac-similé, et les problèmes pratiques reliés à la conservation des livres rares, des journaux et des ouvrages modernes devront être abordés.

La formation des bibliothécaires et autres spécialistes de la documentation, l'orientation philosophique de la profession et l'éducation permanente en bibliothéconomie feront l'objet d'études de la part des membres de la Division de l'éducation et de la recherche.

Enfin, la Division du développement régional, qui a déjà organisé une série de séminaires en collaboration avec l'Unesco, continuera à étudier le problème de la planification et de la mise en place de systèmes adaptés aux besoins des pays en voie de développement, tout en s'intéressant aux thèmes abordés par les autres divisions.

De façon globale, l'UAP augmentera le nombre de prêts entre bibliothèques, tant sur le plan national qu'international, et il est recommandé que l'IFLA élabore un code pour le prêt international. II sera nécessaire de s'occuper du développement rationnel des collections à l'intérieur d'un pays ou d'une zone géographique. Le programme UAP polarisera ainsi les efforts axés sur une amélioration de l'infrastructure des services d'information, dans la perspective de la satisfaction complète de l'usager. 


\section{Le système}

A l'instar du CBU, I'UAP ne peut se réaliser que sur la base d'un réseau de services nationaux intégrés sur le plan international. Selon le même principe adopté par l'Unesco dans le cadre du programme NATIS et du système UNISIST, chaque pays devra prendre les mesures qui lui conviennent pour créer une infrastructure de services de bibliothèques, de documentation et d'archives selon sa propre politique globale de l'information.

Toute la chaîne documentaire y passe, depuis la publication d'un ouvrage, le dépôt légal, le contrôle bibliographique, la rédaction de la bibliographie nationale, jusqu'aux mécanismes d'indexation et au prêt, en passant par l'acquisition de la production d'autres pays par achat, échange ou prêt, pour arriver à l'information offerte à l'usager.

Dans le système UAP, les petites bibliothèques ont un rôle à jouer, tout comme les bibliothèques régionales et les bibliothèques nationales. De plus, un centre de disponibilité universelle des publications pourrait s'occuper de la normalisation, conseiller les pays sur les moyens à prendre pour mettre en oeuvre leurs politiques, mesurer les progrès accomplis et coopérer avec l'Unesco.

En somme, le concept de l'UAP se situe dans le sillage du Contrôle bibliographique universel et l'enrichit, puisqu'il situe l'utilisateur au coeur même de l'élaboration du système. Information et documentation doivent toujours demeurer au service des besoins des usagers. L'UAP ne s'occupe pas seulement de fournir rapidement des renseignements bibliographiques sûrs. Conçu pour l'usager, ce programme lui permettra de découvrir et de consulter les ouvrages qui pourraient l'intéresser.

Au moyen de la Disponibilité universelle des publications et du Contrôle bibliographique universel, I'IFLA se dirige vers ce que son ancien président, Herman Liebaers, a appelé «l'accès à l'information». L'UAP s'adresse à tous ceux qui sont engagés dans les services d'information, pour qu'ils puissent rendre l'information accessible à toutes les catégories d'usagers, dans tous les genres de bibliothèques, dans les pays industrialisés et les pays en voie de développement, et qu'ils arrivent à satisfaire les besoins de leurs utilisateurs.

Le président de la Fédération, Preben Kirkegaard, a adressé aux congressistes réunis à Strbské Pleso en août 1978 un vibrant appel à la coopération afin que le programme atteigne ses objectifs:

We cannot and will not confine our programmes to a torrent of empty words, building up a pitifully fragile facade with no substance behind it. No, what we aim at is the building up of a safe and dependable edifice, an IFLA in which the many types of libraries and library activities work together to achieve realistic international programmes which further national library development and contribute toward improvement of library services for every reader wherever he or she lives ${ }^{10}$.

\section{Bibliographie sur I'UAP}

Allardyce, A. "Some opportunities for IFLA's Division of Collections and Services", IFLA journal, vol. 3, no. 3 (1977), 243-246.

Allardyce, A. "UAP and the exchange of publications», IFLA journal, vol. 4, no. 2 (1978), 122-128.

«Après le congrès de I'I.F.L.A. à Bruxelles", Le Bulletin du livre, no 327 (3 octobre 1977), 90-92.

Bansa, Dr. Helmut. Conservation as a Special Aspect of UAP. Paper presented at the 44th IFLA Congress, Strbské Pleso, 1978. $3 \mathrm{p}$. (19/MAN/2E).

10. Preben Kirkegaard, Opening Speech: Abstracts. Presidential address to the 44th IFLA Congress, Strbské Pleso, 1978, p. 1-2 (50/PLE/5E). 
Clarke, T.C. «Knowing your universals: UAP in relation to UBC», IFLA journal, vol. 4, no. 2 (1978), 129-133.

Genzel, Peper. Universal Availability of Publications and the Section on the Exchange of Publications: Report on its Activities, 1970-1978. Paper presented at the 44th IFLA Congress, Strbské Pleso, 1978. $14 \mathrm{p}$. (28/COLL/2E).

Harbo, Ole. Universal Availability of Publications and Library Research. Paper presented at the 44th IFLA Congress, Strbské Pleso, 1978. $5 \mathrm{p}$. (21/EDU/2E).

Honoré, Suzanne. "Le réseau UAP et les publications officielles», IFLA journal, vol. 4, no. 2 (1978), 134-139.

Kalajdzieva, K. «UAP (Universal Availability of Publications) and its relevance to national libraries from the viewpoint of a small country", IFLA journal, vol. 4, no. 2 (1978), 140-145.

Kalajdzieva, K. L'UAP et son rapport avec les bibliothèques nationales: le point de vue d'un petit pays. Communication présentée au $44^{e}$ congrès de I'IFLA, Strbské Pleso, 1978. 9 p. (1/GEN R/1F).

Lajeunesse, Marcel. «Rapport du Congrès annuel de l'IFLA par le délégué de l'ASTED", Nouvelles de l'ASTED, 1978.

Liebaers, Herman. «Universal Availability of Publications; a concept and a programme», IFLA journal, vol. 4, no. 2 (1978), 117.

Line, Maurice B. «UAP and interlibrary lending", IFLA journal, vol. 4, no. 2 (1978), 118-121.

Line, Maurice B. Universal Availability of Publications: Progress Report, presented at the 44th IFLA Congress, Strbské Pleso, 1978. $3 \mathrm{p}$.

(62/PLE/6E).
Line, Maurice B. «Universal Availability of Publications and developing countries", in IFLA Pre-session Seminar for Developing Countries, Antwerp, Belgium, 1977. The Impact of Computerized Systems and Networks in Resource Sharing of Libraries. Antwerp, Antwerp University, 1977.

Nyarka, Kwame. Universal Availability of Publications (UAP) and Library Education in Africa. Paper presented at the 44th IFLA Congress, Strbské Pleso, 1978. 12 p. (17/EDU/1E).

Rodriguez, Adolfo. L'accès universel aux publications dans les pays en voie de développement. Communication présentée au 43e congrès de l'IFLA, Bruxelles, 1977.6 p. (38/F/IL/3-43F).

Rodriguez, Adolfo. «Universal Availability of Publications in developing countries», Interlending review, vol. 6, no. 3 (July 1978), 90-92.

Soosai, J.S. «Universal Availability of Publications: a third world perspective", IFLA journal, vol. 4, no. 2 (1978), 146-150.

Spicer, Erik J. "Universal Availability of Publications (UAP) and parliamentary libraries», IFLA journal, vol. 4, no. 2 (1978), 151-154.

Tuillier, André. L'importance de la formation scientifique du bibliothécaire dans l'accès universel aux documents. Communication présentée au $44^{e}$ congrès de l'IFLA, Strbské Pleso, 1978, 3 p. (29/TRAIN/2F).

Urquhart, D.J. Quelques aspects de l'A.U.P. dans un pays développé. Communication présentée au $43^{e}$ congrès de I'IFLA, Bruxelles, 1977. 4 p. $(32 / F / I F / 1)$.

Urquhart, D.J. L'UAP: quelles mesures faut-il prendre? Communication présentée au 44 e congrès de l'IFLA, Strbské Pleso, 1978. $14 \mathrm{p}$. (5/COLL/1F). 
Van Swigchem, P.J. "UAP: a task for libraries serving the general public", IFLA journal, vol. 4, no. 2 (1978), 155-157.

Vickers, Stephen. Vers un système mondial d'A.U.P. Communication présentée au $43^{\circ}$ congrès de l'IFLA, Bruxelles, 1977. 9 p. (33/IL/2F).

Willison, Ian R. «The relevance of Universal Availability of Publications to rare and precious books», IFLA journal, vol. 4 , no. 2 (1978), 158-165.

\begin{abstract}
A noter:
Une série d'articles sur l'UAP dans divers pays doit paraître dans le deuxième numéro du Bulletin de l'Unesco à l'intention des bibliothèques en 1979.

On peut se procurer les communications présentées aux congrès de l'IFLA en s'adressant au Centre de documentation sur les bibliothèques, 395, rue Wellington, Ottawa K1A ON4.
\end{abstract}

\section{DIRECTEUR BIBLIOTHĖQUE PUBLIQUE D'OTTAWA}

Le Conseil d'administration de la Bibliothèque publique d'Ottawa cherche à combler le poste de Directeur de la bibliothèque et de secrétaire-trésorier du Conseil d'administration par un bibliothécaire professionnel d'expérience. Le titulaire entrera en fonctions vers le $1^{\text {er }}$ novembre 1979.

Capitale nationale, la ville d'Ottawa incarne une intéressante communauté bilingue dotée de nombreux services gouvernementaux, universitaires, culturels et récréatifs. Pour desservir cette communauté, la Bibliothèque publique d'Ottawa dispose d'une collection de plus de 600,000 volumes dont une importante proportion est en français. L'organisme comporte une nouvelle bibliothèque centrale, sept succursales, trois bibliobus et un personnel de 188 employés à plein temps. Ses crédits annuels se chiffrent par près de cinq millions de dollars et le nombre de prêts dépasse annuellement les deux millions d'articles. La bibliothèque souscrit au système informatisé UNICAT/TELECAT et, par ailleurs, envisage aussi d'automatiser son catalogue et ses procédures de contrôle du prêt.

Le Directeur, appuyé du Directeur adjoint et de l'Agent d'administration, est comptable au Conseil d'administration. Le Directeur aide à élaborer objectifs et politiques, exécute les décisions du Conseil, dirige et coordonne les activités du personnel en vue d'atteindre les objectifs fixés. Le Directeur est responsable de l'administration financière de la bibliothèque. A ce titre, il doit en planifier le budget, le présenter au Conseil municipal et gérer les ressources financières qui y sont consacrées.

Le dossier du titulaire devra témoigner d'importants succès progressifs tant dans le domaine professionnel que dans la gestion d'une bibliothèque aux multiples services. Des aptitudes en administration et en communication, de l'expérience en relations publiques et humaines, de l'enthousiasme et une bonne santé sont des qualités que recherche, en l'occurence, le Conseil d'administration. Enfin, le bilinguisme dans les deux langues officielles ainsi que la connaissance et la compréhension des cultures qu'elles représentent, sont des atouts importants.

Traitement pour 1978: $\$ 36,236$ - $\$ 43,365$ (En voie de révision).

Les candidatures, étayées de références, doivent être déposées avant le 31 mars 1979 à l'adresse suivante:

Le Président

Comité de recherche

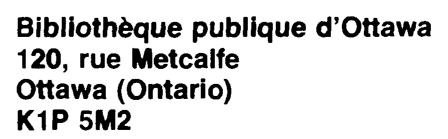

